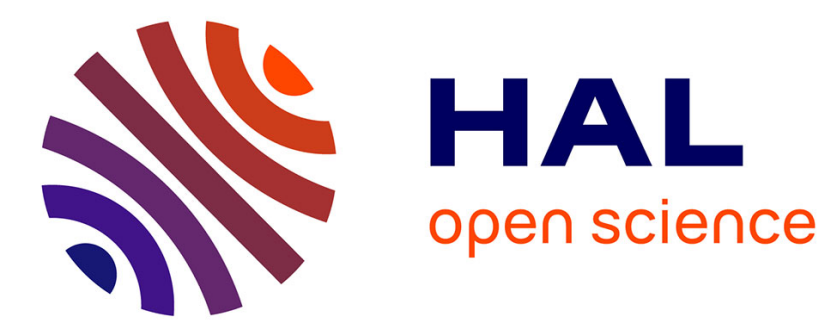

\title{
Studying the pulse regime in a reverberation chamber with a model based on image theory
}

Emmanuel Amador, Christophe Lemoine, Philippe Besnier, Alexandre Laisné

\section{To cite this version:}

Emmanuel Amador, Christophe Lemoine, Philippe Besnier, Alexandre Laisné. Studying the pulse regime in a reverberation chamber with a model based on image theory. IEEE International Symposium on EMC 2010, Jul 2010, Fort Lauderdale, United States. pp.520-525, 10.1109/ISEMC.2010.5711330 . hal-00514254

\section{HAL Id: hal-00514254 \\ https://hal.science/hal-00514254}

Submitted on 1 Sep 2010

HAL is a multi-disciplinary open access archive for the deposit and dissemination of scientific research documents, whether they are published or not. The documents may come from teaching and research institutions in France or abroad, or from public or private research centers.
L'archive ouverte pluridisciplinaire HAL, est destinée au dépôt et à la diffusion de documents scientifiques de niveau recherche, publiés ou non, émanant des établissements d'enseignement et de recherche français ou étrangers, des laboratoires publics ou privés. 


\title{
Studying the Pulse Regime in a Reverberation Chamber with a Model Based on Image Theory
}

\author{
Emmanuel Amador ${ }^{\# 1}$, Christophe Lemoine *, Philippe Besnier \#, Alexandre Laisné * \\ \# IETR, INSA Rennes \\ 20 Av. des Buttes de Coësmes, 35708 Rennes, FRANCE \\ 1 emmanuel.amadordinsa-rennes. fr \\ ${ }^{*}$ CEAT, DGA-ATU \\ 47 rue Saint Jean, 31130 Balma, FRANCE
}

\begin{abstract}
In this article, we propose a time-domain model of a reverberation chamber ( $\mathrm{RC})$. This very straightforward model based upon image theory (IT) allows to simulate the behavior of a reverberation chamber in the pulse regime. After a brief introduction of the model, results with different pulse lengths and different loading configurations are presented and compared to measurements made in the reverberation chamber of our laboratory.
\end{abstract}

\section{INTRODUCTION}

\section{A. Context}

Reverberation chambers (RC) are traditionally used and studied in continuous wave $(\mathrm{CW})$ and in frequency domain to conduct electromagnetic compatibility (EMC) measurements. Performing an immunity test in $\mathrm{CW}$ is not relevant if the device under test (DUT) receives radar signals. In order to study the immunity of devices with radar-like signals, the behavior of the $\mathrm{RC}$ in time domain and in pulse regime must be studied carefully. Few works are available today to understand the phenomena involved in the pulse regime in a RC. In [1] and [2], the authors performed immunity testing with a real radar source. Waveforms and levels obtained with different loading are discussed. Transients in an RC have also been investigated in mode-tuned RCs [3]. Because of the transients, the waveforms obtained in the pulse regime can be very disparate and parameters like the pulsed signals used, the gain and the positions of the antennas and the DUT in the RC can change drastically the waveforms and the levels obtained in the chamber. Studying an RC in time-domain means that we have to control more parameters and acquire more data than in a frequency-domain analysis. A model able to simulate a $\mathrm{RC}$ in time-domain can be helpful to understand roughly the influence of each parameter on the levels obtained and the waveforms recorded.

\section{B. Time Domain Models of Reverberation Chambers}

The solutions to make time-domain simulations of RCs are based on transmission line matrix (TLM) [4] or finite differences time domain (FDTD) [5], [6], [7]. These methods use a space-time discretization of an electrically large and highly conductive environment and are complicated to perform. Optical techniques like ray tracing (RT) are not able to simulate hundreds of reflections and so cannot reproduce the reverberation phenomenon in the chamber. There is a real need for a straightforward model of an RC able to simulate the physics of the RC through some parameters like its dimensions, the loading in the chamber, and the waveform used. After a brief presentation of the model we developed, we will compare various results obtained by our model with measurements made in the RC of our laboratory. Levels and waveforms obtained are compared for different loading configurations with a short pulse. Statistical analyses of the E-field measured and simulated during longer pulses are given from the point of view of immunity testing.

\section{Model of A Reverberation Chamber with Image THEORY}

\section{A. Cavity Modeling Using Image Theory}

In [8], the author presents a model of a waveguide based on image theory (IT). By adding two boundary conditions, we can easily use this approach to model a shielded rectangular cavity. In a paper published in 1998 [9], the author uses IT to model a rectangular shielded cavity. The application of the model is limited to an asymptotic extraction of the quality factor. To our knowledge it is the first paper about a time-domain analysis of a shielded cavity based upon an optical model. Few years later, in another paper [10], the author investigates various approaches to model an RC. A 2D and a 3D model based on IT are proposed, but the computer resources did not allow to get relevant results.

\section{B. Presentation of our model}

Our model is based upon IT [8]. If we assume that the walls of our shielded cavity are mirrors and our emitting antenna is a lightbulb, we have to determine with accuracy the position of every image of the lightbulb in this optical cavity to reproduce the reflections by the six walls. If our antenna is an elementary current, we have to determine the orientation of every image-current created by the reflective walls (Fig.1). This very straightforward approach does not involve directly the Maxwell's equations nor a spatial discretization of the environment, but it is enough to carry the physics of an RC. 


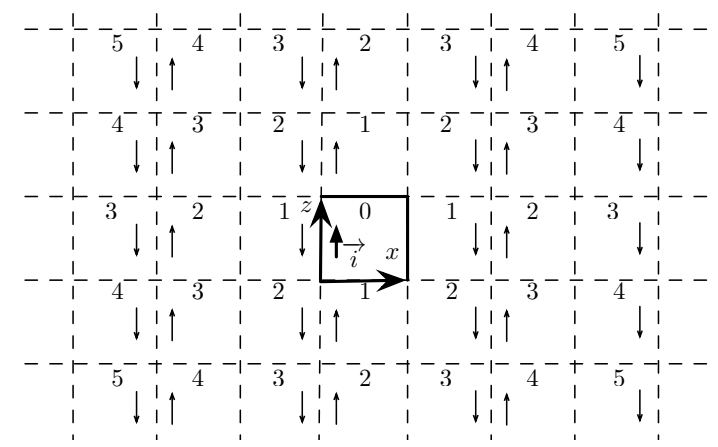

Fig. 1. Image currents in a vertical plane. Orders are mentioned in each cavity.

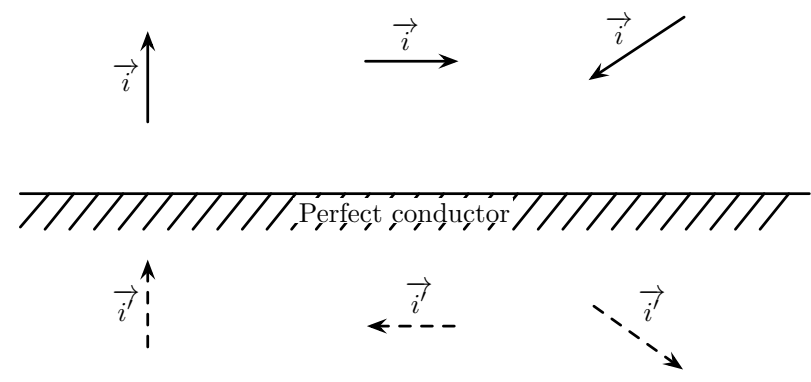

Fig. 2. Image theory applied to currents.

Our model computes a channel impulse response (CIR). The CIR can be convoluted with a chosen waveform to obtain the response of the cavity with this signal. A Fourier transform of the CIR allows to explore the frequency domain. Our model does not include a stirrer and so cannot be deterministic. However, the analysis of the results is statistical. It consists in a Monte Carlo method that chooses randomly a number of points in the volume of the chamber. The results obtained with this model are analyzed and confronted statistically with measurements made in the RC at the Institute of Electronics and Telecommunications of Rennes (IETR).

\section{Image Theory Model}

1) Image creation: IT is generally introduced with electric charges and currents [8]. Fig. 2 sums up the different possible configurations with an electric current vector $\vec{i}$ and a infinite perfectly conducting plane. Figure 1 inspired by [8] presents a vertical view of the image currents created by applying IT to a current oriented along the $O z$ axis of a rectangular cavity. The real cavity (in bold line, in the middle) is surrounded by image cavities. Each image cavity contains an image current. The first step is to determine the position and the orientation of every elementary image current. This process is accelerated by identifying patterns and similarities. We define the order of an image current as the number of reflections involved in its creation. The number of cavities for a given order $n$ is given by:

$$
N_{n}=4 n^{2}+2
$$

and the total number of cavities till the order $n$ is given by:

$$
\begin{aligned}
M_{n} & =1+\sum_{i=1}^{n}\left(4 i^{2}+2\right) \\
& =1+2 n+\frac{2 n(n+1)(2 n+1)}{3} .
\end{aligned}
$$

The growth of $M_{n}$ is proportional to $n^{3}$. The dimensions of the RC in our laboratory are $8.7 \times 3.7 \times 2.9 \mathrm{~m}$. In order to simulate the first microsecond, the number of currents involved is around 8 millions. For the first 3 microseconds, the number of currents reaches 230 millions. On a 64 bits platform with $32 \mathrm{~GB}$ of memory we managed to simulate the first 3 microseconds in our cavity.

2) Cavity loss: To simulate a lossy rectangular cavity, we introduce three loss coefficients $R_{x}, R_{y}, R_{z}$ corresponding to the three pairs of conducting walls of our cavity (the distinction of the three directions can be helpful if the materials used for the walls are different). In this section we focus on an elementary current $a$ inside a $n^{\text {th }}$ order image-cavity. This elementary current is created by $i$ reflections along the $O x$ axis, $j$ reflections along the $O y$ axis and $k$ reflections along the $\mathrm{O} z$ axis equals. The attenuation associated to this elementary current $a$ equals:

$$
R_{a}=R_{x}^{i} R_{y}^{j} R_{z}^{k}, \text { with } i+j+k=n
$$

and the intensity of this current will be written:

$$
I_{a}=I_{0} \cdot R_{a},
$$

where $I_{0}$ is the intensity of every current in the system if the walls are perfectly conductive. The total amount of energy found in the system is proportional to (if we pose $R=R_{x}=$ $\left.R_{y}=R_{z}\right)$ :

$$
E_{t o t} \propto I_{0}^{2}+\sum_{i=1}^{\infty}\left(4 i^{2}+2\right) I_{0}^{2} \cdot R^{2 i}
$$

As $R<1$, the sum above converges.

3) Channel impulse response simulation: If the current in the real cavity is oriented along the $O z$ axis. All the image currents are oriented along $O z$. The current $\overrightarrow{I_{a}}$ of the particular elementary current $a$ can be written:

$$
\overrightarrow{I_{a}}=(-1)^{i+j} I_{a} \cdot \overrightarrow{e_{z}}=(-1)^{i+j} I_{0} R_{x}^{i} R_{y}^{j} R_{z}^{k} \cdot \overrightarrow{e_{z}}
$$

The current in the real cavity and all the image currents emit simultaneously an elementary impulse $f(t)$. The intensity $I_{a}$ of the elementary current $a$ can be written:

$$
I_{a}(t)=(-1)^{i+j} I_{0} R_{a} \cdot f(t)
$$

In the spherical coordinates attached to this particular current, the E-field created by the elementary current $a$, received at a given reception point $P$ within the real cavity can be written: $\overrightarrow{E_{a}}(t)=-E_{a}(t) \sin \theta_{a}\left(\cos \phi_{a} \cdot \overrightarrow{e_{x}}+\sin \phi_{a} \cdot \overrightarrow{e_{y}}+\sin \theta_{a} \cdot \overrightarrow{e_{z}}\right)$,

with:

$$
E_{a}(t)=\omega \mu \frac{(-1)^{i+j} d h I_{0} R_{a}}{d_{a}} f\left(t-\frac{d_{a}}{c}\right)
$$


(a)

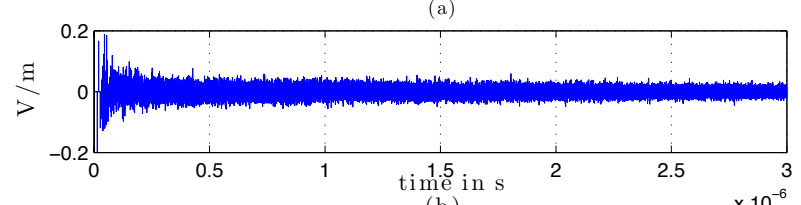

(b)
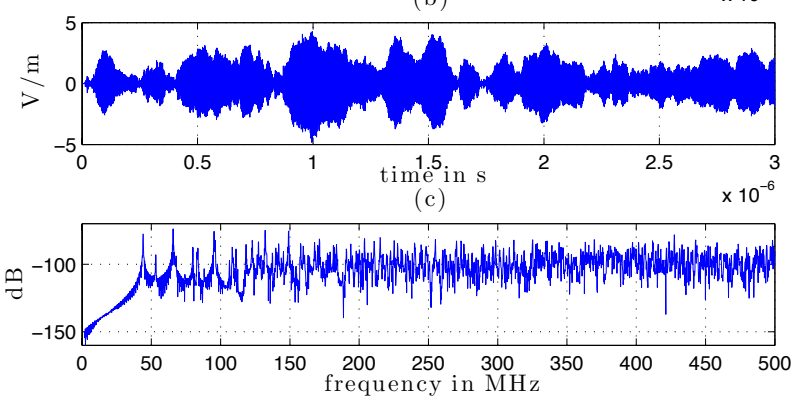

Fig. 3. (a) Channel impulse response ( $\mathrm{E}_{z}$ component), (b) Response for a $300 \mathrm{~ns}$ long pulse at $1 \mathrm{GHz}\left(\mathrm{E}_{z}\right.$ component), (c) Frequency response $\left(\mathrm{E}_{z}\right.$ component).

where $d_{a}$ is the distance between the position of the elementary current $a$ and the reception point $P, \theta_{a}$ and $\phi_{a}$ are the angular coordinates of the point $P$ in the local coordinate system attached to the elementary current $a$ and $c$ the speed of light.

The CIR is given by adding the contribution of every current in our system. If $M$ is the number of currents in our system, from (9) we can deduce three channel impulse $\left(s_{x}, s_{y}, s_{z}\right)$ responses corresponding to the three rectangular components:

$$
s_{x, y, z}(t)=\sum_{i=0}^{M} \vec{E}_{i}(t) \cdot \overrightarrow{e_{x, y, z}}
$$

In the general case, the current inside the real cavity is oriented arbitrary with given elevation and azimuth angles. Hence, each image-current is oriented with particular elevation and azimuth angles. The calculation of the impulse responses involves a coordinate transformation of the reception point from the local spherical system of coordinates associated with the considered image current to the common rectangular coordinates associated with the cavity walls. This feature is implemented in the model but not presented here to clarify this article.

The loss coefficient $R$ can be determined empirically by adjusting gradually the loss coefficient in the model to match the power delay profile measured in the RC. Generally, we use $R=0.998$ to simulate an empty cavity. With $R=0.97$ we can consider that the chamber is heavily loaded.

\section{Preliminary Results}

In this section we present some results in time domain and frequency domain. Let $A$ be the position of the emitting elementary current and $B$ the position of the reception point. Figure 3-(a) shows the CIR along the vertical component $\left(E_{z}\right)$ obtained by our model with $R_{x}=R_{y}=R_{z}=0.998$, we can note that the pulse amplitude is slowly decreasing. One can imagine that the power delay profile of such a situation would be very long. After a convolution of this signal with

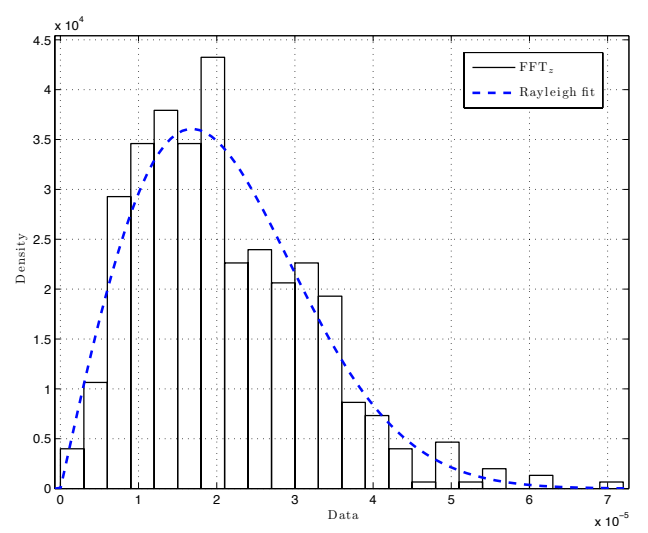

Fig. 4. Probability density function of $\operatorname{FFT}\left(E_{z}\right)$ over $100 \mathrm{MHz}$ around 1950 $\mathrm{MHz}$ (500 samples).

a 300 ns long pulse at $1 \mathrm{GHz}$, we obtain the response of the cavity for this signal (Fig. 3-(b)). Figure 3-(c) shows the frequency response of the channel obtained by applying a Fourier transform on the CIR. The frequency response clearly exhibits the resonant frequencies one should expect for a cavity of these dimensions $(8.7 \times 3.7 \times 2.9 \mathrm{~m})$. After $400 \mathrm{MHz}$, the Fourier transform exhibits fast fading. A quick analysis of the distribution of the $E_{z}$ component between $1900 \mathrm{MHz}$ and 2000 $\mathrm{MHz}$ over 500 independent frequencies [11] shows clearly that the rectangular component $E_{z}$ follows a Rayleigh distribution [12] (Fig. 4).

In order to visualize the cavity modes involved for a given frequency and to verify that the physics of resonant cavities is respected we perform a simulation into a full horizontal plane of the cavity. Figure 5 shows the result obtained in a full plane after a Fourier transform of the vertical component $\left(E_{z}\right)$ for two different frequencies. These results show clearly that the modes excited at a given frequency match the modes theoretically present in the cavity. The geometry of the modes is respected and the boundary conditions that state that the tangential components of the E-field are null on the conductive walls are respected.

These results show that in spite of its simplicity, the model carries the physics of an RC and its frequency domain behavior is very similar to a real cavity.

\section{Pulse Regime Simulations of a Real REVERBERATION CHAMBER, LEVELS AND WAVEFORMS WITH DiFFERENT LOADING CONFIGURATIONS}

The purpose of this section is to show how the model can predict the levels obtained in a chamber for a given signal and a given loading.

\section{A. Simulation of a Pulse in a Plane}

Figure 6 shows the propagation of a $100 \mathrm{~ns}$ pulse at $500 \mathrm{MHz}$ in an horizontal plane. We can note that the reflections on the walls are respected and that the energy is scattered randomly in the plane. The loss coefficient $R=0.98$, means that the power in the chamber decays rapidly after the end of the pulse. 

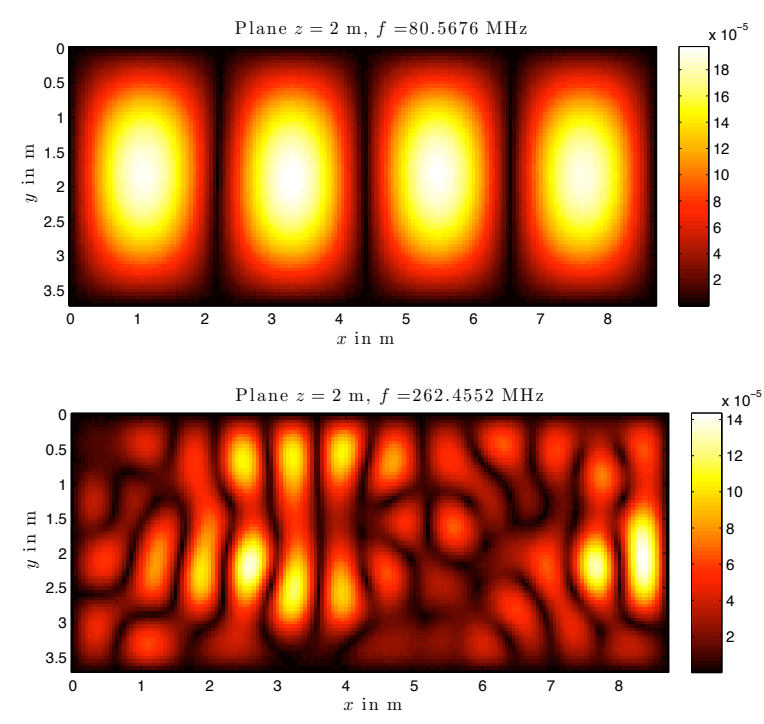

Fig. 5. Fourier transform of the $E_{z}$ component of the electric field in the plane $z=2 \mathrm{~m}$ at different frequencies. The $\mathrm{TE}_{410}$ cavity mode (top) and a combination of different cavity modes around the LUF (bottom) are presented. Linear values.
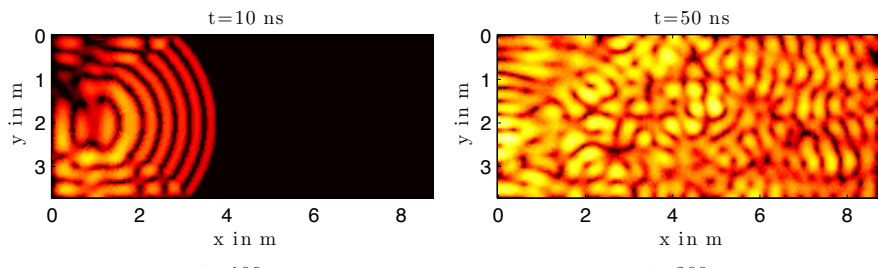

$\mathrm{t}=100 \mathrm{~ns}$
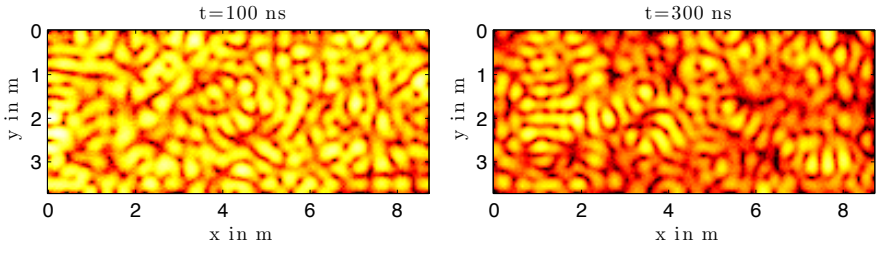

$\mathrm{t}=500 \mathrm{~ns}$
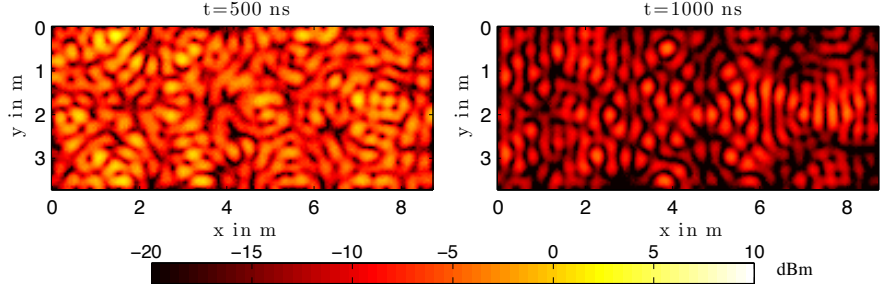

Fig. 6. Simulation of the propagation of a $100 \mathrm{~ns}$ pulse at $500 \mathrm{MHz}$ in an horizontal plane with $R=R_{x}=R_{y}=R_{z}=0.98$, total power in $\mathrm{dBm}$.

\section{B. Simulation of a 300 ns Long Pulse with Different Loading Configurations}

To conduct an immunity testing, one should know the mean and the maximum levels a DUT will receive during the test [13]. We want to know if our model is able to predict the levels and the average waveforms for a given loading and a given signal. The emitting antenna and the DUT are both wide-band discone antennas (Fig. 7).

1) Empty RC: The first step is to measure an impulse channel response. The length of the channel impulse response

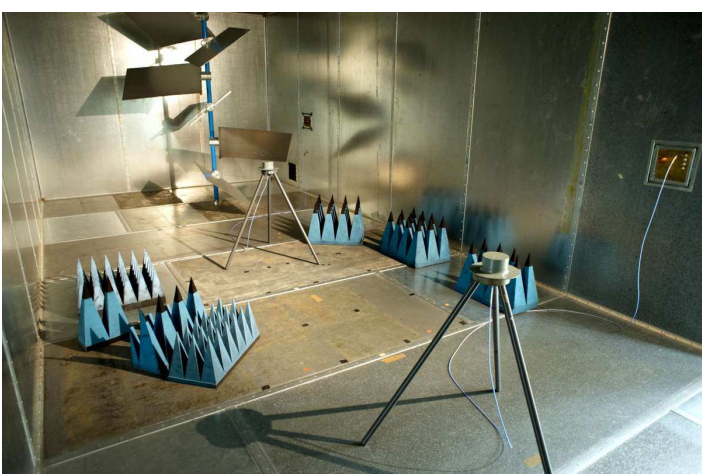

Fig. 7. Experimental configuration with loading.

(a)
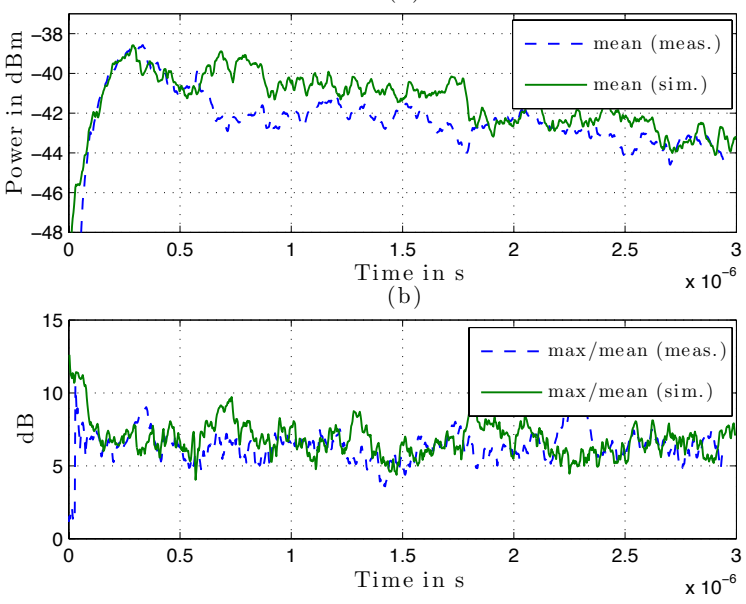

Fig. 8. Mean power from measurements and simulations (a), maximum power/mean power ratios from measurements and simulations (b) for a 300 ns long pulse at $1 \mathrm{GHz}$ in an empty RC.

gives an estimation of the loss coefficient used in the model. We found $R=R_{x}=R_{y}=R_{z}=0.998$. The pulse signal $(\tau=300 \mathrm{~ns}$ at $1 \mathrm{GHz})$ is emitted. The receiving antenna is moved in the RC. We made 50 measurements with a digital storage oscilloscope (DSO). The situation is reproduced in the model, by calculating 50 simulations at 50 arbitrary independent positions [14] in our virtual cavity. From both the measurements and the simulations we extract the average of the levels received and the max for every time-step, the levels obtained by simulations in $\mathrm{dB}$ are corrected by adding a constant bias to fit the measurements. Figure 8-(a) shows a great adequacy between the measurements and the simulations. Figure 8-(b) shows that the ratio max/mean is around $6.5 \mathrm{~dB}$ in both the simulations and the measurements [15], [16].

2) With loading: Our RC is now loaded with absorbers (Fig. 7). The loss coefficient is now $R=0.975$. Figure 9 shows that the results obtained with our model follows the measurements made in the loaded cavity. The waveforms obtained are very similar, the maximum deviation between the simulations and the measurements is around $3 \mathrm{~dB}$. The ratio $\max /$ mean is around $6.5 \mathrm{~dB}$ in both the simulations and the measurements. 
(a)

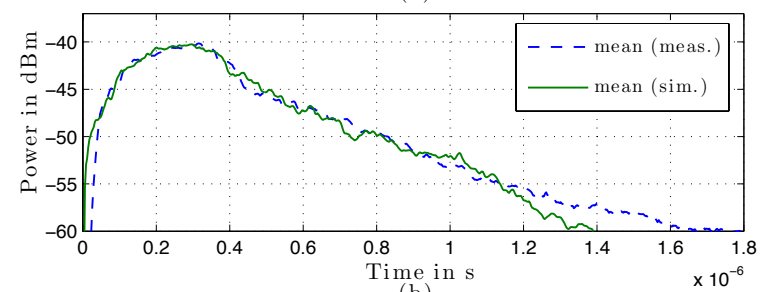

(b)

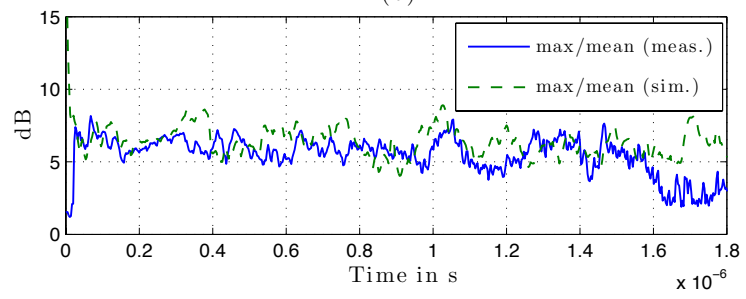

Fig. 9. Mean power from measurements and simulations (a), maximum power/mean power ratios from measurements and simulations (b) for a 300 ns long pulse at $1 \mathrm{GHz}$ in a loaded $\mathrm{RC}$.

The model presented in this article is able to simulate with great accuracy the levels obtained for a given loading and a given waveform. It will be helpful to predict the levels in the pulse regime in order to perform immunity testing with pulsed signals. In the next section, the statistical aspects of the E-field are analyzed with longer pulses.

\section{Statistical Analysis of the Electric Field in TIME DOMAIN, EFFECT OF THE LOADING}

\section{A. Modus Operandi}

This section presents an analysis of the distribution followed by the E-field of a rectangular component as a function of time. When the $\mathrm{RC}$ is used in continuous wave $(\mathrm{CW})$ mode and beyond the lowest usable frequency, we know that for a given position, the component of the E-field follows a Rayleigh distribution over a complete rotation of the stirrer. The Rayleigh distribution assures the statistical homogeneity of the E-field in the chamber. Generally immunity testing is performed in frequency domain and $\mathrm{CW}$ and the duration of the exposure is sufficiently long to reach the continuous regime. If the immunity testing is performed in time domain it can be interesting to study the statistical properties of the E-field during the transients. A $1 \mu$ s pulse at $1 \mathrm{GHz}$ is emitted in the chamber. The signal is recorded with a DSO. The antennas are two wide band horn antennas placed in the chamber to limit the unstirred component. We choose $N=100$ stirrer positions. From the $N$ waveforms recorded, we extract their envelope. For every instant we obtain $N$ measurements of the amplitude recorded by the DSO. The amplitude recorded is directly linked to the incident E-field vertical component on the receiving antenna. In our model, the stirring process is assured by choosing $N=100$ independent positions randomly in the volume of the chamber. For every time step, the corresponding $N$-size sample is tested with the Anderson Darling (AD) goodness of fit test [12] with Stephen's values. The null hypothesis $H_{0}$ is accepted when
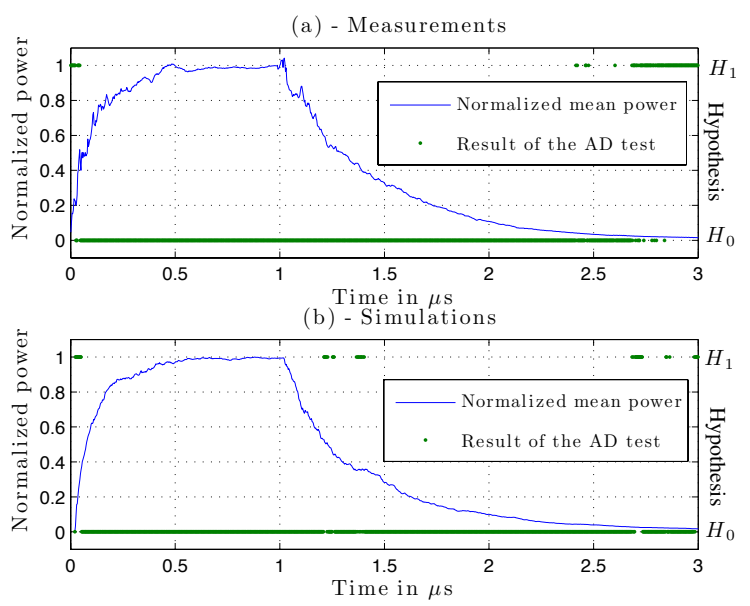

Fig. 10. Mean power and result of the AD test from measurements (a) and simulations (b), for a $1 \mu$ s long pulse at $1 \mathrm{GHz}$ in a loaded $\mathrm{RC}(R=0.97)$.

the population constituted of the $N$ observations follows a Rayleigh distribution. On the contrary the alternative hypothesis $H_{1}$ favors that the distribution is not Rayleigh distributed. The level of significance $\alpha=0.01$ means that the test will fail to recognize $1 \%$ of the Rayleigh distributions.

\section{B. $1 \mu$ s pulse at $1 \mathrm{GHz}$ with and without loading}

Figure 10-(a) shows the average power (normalized linear values) and the result of the $\mathrm{AD}$ test obtained with measurements in the RC. In this situation, the chamber is heavily loaded and the rise time is very short in this situation (around $500 \mathrm{~ns}$ ). Figure 10-(b) shows the corresponding simulation with the model. The chamber is loaded with absorbers and the loss coefficient $R$ is 0.97 in the simulations. The loading and the length of the pulse allow to reach the permanent regime after 500 ns. Both the average power simulated in this situation and the results of the AD test are very similar to the measurements. These results show that a component of the E-field in our RC follows a Rayleigh distribution after $50 \mathrm{~ns}$ (best seen in figure 11). If we look carefully at the propagation of the pulse in an horizontal plane (Fig. 6), we can notice that after $50 \mathrm{~ns}$, the power starts to be homogeneously scattered in the cavity. It means that even during the transients, the distribution followed by an E-field component inside an RC follows a Rayleigh distribution. $50 \mathrm{~ns}$ is almost the time needed for the signal to travel twice the length of the chamber. These results show that the homogeneity of the field in the RC is reached rapidly.

Figure 12 shows the same experiment with an empty RC. We can notice that the E-field in the chamber follows a Rayleigh distribution very early. The rise time is longer and with a $1 \mu \mathrm{s}$ long pulse, the permanent regime is not reached.

These results show that the model allows to simulate the waveforms obtained for a rectangular cavity, a given loading and an arbitrary signal and gives access to the 


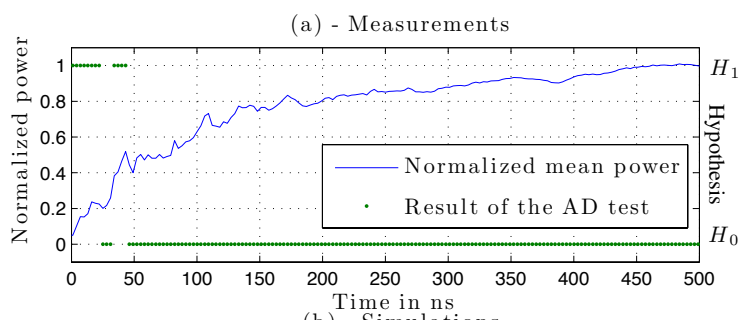

(b) - Simulations

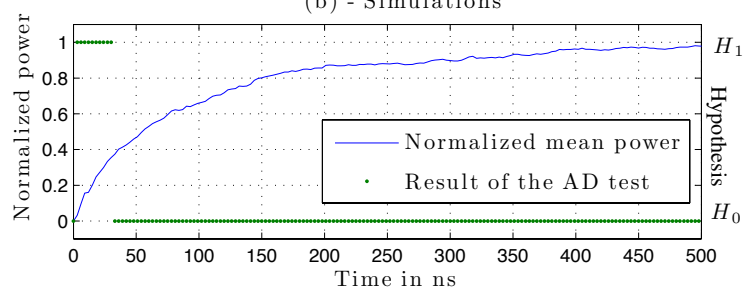

Fig. 11. Focus on the first 500 ns: mean power and result of the AD test from measurements (a) and simulations (b), for a $1 \mu$ s long pulse at $1 \mathrm{GHz}$ in a loaded RC $(R=0.97)$.
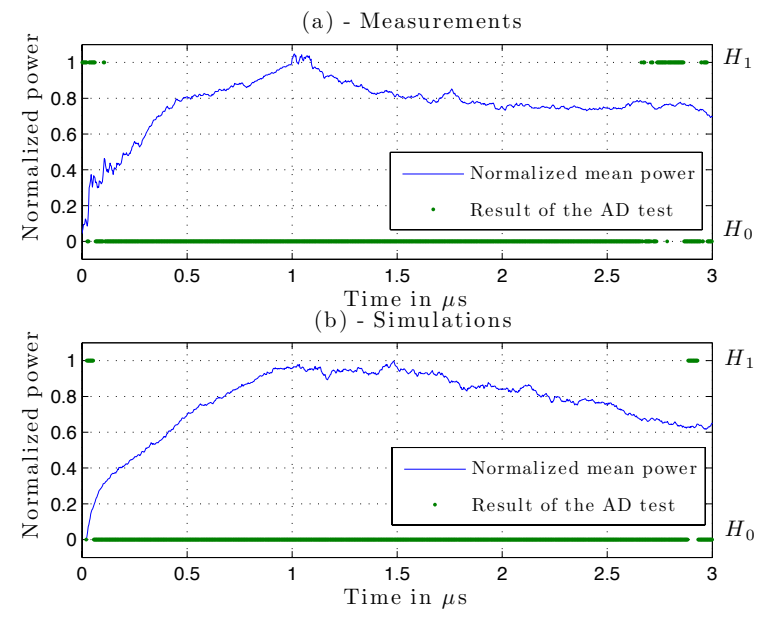

Fig. 12. Mean power and result of the AD test from measurements (a) and simulations (b), for a $1 \mu$ s long pulse at $1 \mathrm{GHz}$ in an empty $\mathrm{RC}(R=0.998)$.

statistics of the field in the RC with a great accuracy. This model allows to estimate the amount of energy received by a DUT during a pulse. This model can be useful to setup and optimize an immunity testing in the pulse regime. With the E-field received by a DUT during a pulse, we can easily extract the induced currents and quantify the stress created on a circuit during a pulse.

\section{CONClusion}

The results presented in this article show that with few physical considerations, we can predict precisely the behavior of an RC in time domain. The model presented can be considered as the simplest model possible of an RC that carry the physics of the cavity (frequency response and cavity loss). This model is able to simulate the behavior of an RC in time domain and to predict the levels obtained in a cavity for a given signal and a given loading. It may be precious to study and understand the physical phenomena involved in time domain in an RC. From an immunity testing point of view, this model may be helpful to setup and optimize the various parameters of the test.

\section{ACKNOWLEDGMENT}

This work was supported by the French Ministry of Defence DGA (Direction Générale de l'Armement), with a Ph.D. grant delivered to Emmanuel Amador and a "REI" grant 200834004 delivered to IETR (INSA de Rennes). The authors would like to thank Jérôme Sol for his assistance during the measurements.

\section{REFERENCES}

[1] O. Lunden and M. Backstrom, "Pulsed power $3 \mathrm{GHz}$ feasibility study for a $36.7 \mathrm{~m}^{3}$ mode stirred reverberation chamber feasibility study for a $36.7 \mathrm{~m}^{3}$ mode stirred reverberation chamber," in Electromagnetic Compatibility, 2007. EMC 2007. IEEE International Symposium on, July 2007, pp. 1-6.

[2] — , "Absorber loading study in foi $36.7 \mathrm{~m}^{3}$ mode stirred reverberation chamber for pulsed power measurements," in Electromagnetic Compatibility, 2008. EMC 2008. IEEE International Symposium on, Aug. 2008, pp. $1-5$.

[3] L. Arnaut, "Time-domain measurement and analysis of mechanical step transitions in mode-tuned reverberation: Characterization of instantaneous field," Electromagnetic Compatibility, IEEE Transactions on, vol. 49, no. 4, pp. 772-784, Nov. 2007.

[4] A. Coates, H. Sasse, D. Coleby, A. Duffy, and A. Orlandi, "Validation of a three-dimensional transmission line matrix (TLM) model implementation of a mode-stirred reverberation chamber," Electromagnetic Compatibility, IEEE Transactions on, vol. 49, no. 4, pp. 734-744, Nov. 2007.

[5] C. Bruns and R. Vahldieck, "A closer look at reverberation chambers 3-D simulation and experimental verification," Electromagnetic Compatibility, IEEE Transactions on, vol. 47, no. 3, pp. 612-626, Aug. 2005.

[6] M. Höijer, A.-M. Andersson, O. Lunden, and M. Backstrom, "Numerical simulations as a tool for optimizing the geometrical design of reverberation chambers," in Electromagnetic Compatibility, 2000. IEEE International Symposium on, vol. 1, 2000, pp. 1-6.

[7] G. Orjubin, F. Petit, E. Richalot, S. Mengue, and O. Picon, "Cavity losses modeling using lossless FDTD method," Electromagnetic Compatibility, IEEE Transactions on, vol. 48, no. 2, pp. 429-431, May 2006.

[8] R. Harrington, Time-Harmonic Electromagnetic Fields. New York: McGraw-Hill Book Company, 1961, pp. 103-105.

[9] D.-H. Kwon, R. Burkholder, and P. Pathak, "Ray analysis of electromagnetic field build-up and quality factor of electrically large shielded enclosures," Electromagnetic Compatibility, IEEE Transactions on, vol. 40, no. 1, pp. 19-26, Feb 1998.

[10] S. Baranowski, D. Lecointe, M. Cauterman, and B. Demoulin, "Use of 2D models to characterize some features of a mode stirred reverberation chamber," vol. 9-13. International Symposium on Electromagnetic Compatibility, Sorrento, Italy, september 2002, pp. 381-386.

[11] C. Lemoine, P. Besnier, and M. Drissi, "Estimating the effective sample size to select independent measurements in a reverberation chamber," Electromagnetic Compatibility, IEEE Transactions on, vol. 50, no. 2, pp. 227-236, May 2008.

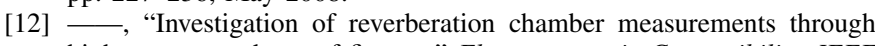
high-power goodness-of-fit tests," Electromagnetic Compatibility, IEEE Transactions on, vol. 49, no. 4, pp. 745-755, Nov. 2007.

[13] "IEC 61000-4-21: Electromagnetic compatibility (EMC) - part 4-21: Testing and measurement techniques - reverberation chamber test methods," IEC, Tech. Rep., 2003.

[14] D. Hill and J. Ladbury, "Spatial-correlation functions of fields and energy density in a reverberation chamber," Electromagnetic Compatibility, IEEE Transactions on, vol. 44, no. 1, pp. 95-101, Feb 2002.

[15] J. Ladbury, G. Koepke, and D. Camell, "Evaluation of the NASA Langley research center mode-stirred chamber facility," NIST, Technical Note 1508, January 1999.

[16] M. Höijer, "Maximum power available to stress onto the critical component in the equipment under test when performing a radiated susceptibility test in the reverberation chamber," Electromagnetic Compatibility, IEEE Transactions on, vol. 48, no. 2, pp. 372 - 384, May 2006. 